\title{
Antiphospholipid Antibodies: Cognitive and Motor Decline, Neuroimaging and Neuropathology
}

\author{
Zoe Arvanitakis $^{a, b}$ Ana W. Capuano ${ }^{a, b}$ Robin Brey ${ }^{c}$ Debra A. Fleischman $^{a, b}$ d \\ Konstantinos Arfanakis ${ }^{a}$, e Aron S. Buchman ${ }^{a} b$ Julie A. Schneider ${ }^{a}, b, f$ \\ Steven R. Levine ${ }^{g}$ David A. Bennett ${ }^{a, b}$ \\ ${ }^{a}$ Rush Alzheimer's Disease Center, Rush University Medical Center, Chicago, IL, USA; ${ }^{b}$ Department of Neurological

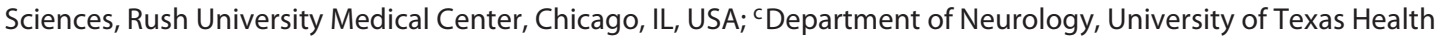 \\ Sciences Center at San Antonio, San Antonio, TX, USA; ${ }^{d}$ Department of Behavioral Sciences, Rush University Medical \\ Center, Chicago, IL, USA; ${ }^{e}$ Department of Biomedical Engineering, Illinois Institute of Technology, Chicago, IL, \\ USA; ${ }^{\mathrm{f}}$ Department of Pathology, Rush University Medical Center, Chicago, IL, USA; ${ }^{9}$ Department of Neurology, \\ State University of New York College of Medicine, Downstate Medical Center, and Kings County Hospital Center, \\ Brooklyn, NY, USA
}

\section{Keywords}

Antiphospholipid antibodies - Cognitive function · Motor function - Magnetic resonance imaging · Neuropathology · Cerebrovascular disease · Aging

\begin{abstract}
Background: Few data are available on associations of antiphospholipid (aPL) antibodies with cognitive and motor decline in aging, and cerebrovascular disease on in vivo neuroimaging and postmortem neuropathology. Methods: This longitudinal, clinical-pathologic study (aPL antibodies, brain infarcts, and cognitive and motor decline in aging), was derived from 2 ongoing community-based cohort studies. A panel of $3 \mathrm{aPL}$ antibodies was assayed in serum from 956 older individuals (mean age $=81.1$ years; $72 \%$ women). Serum was also tested in a subset for markers of inflammation (C-reactive protein [CRP]) and blood-brain barrier break-
\end{abstract}

down (matrix metalloproteinases, MMPs). Annual clinical evaluations documented cognitive (17 neuropsychological tests) and motor function including parkinsonism. Cerebrovascular disease data were derived from in vivo neuroimaging and postmortem neuropathologic evaluations (699 individuals). We examined associations of aPL with cognitive and motor decline, other serum markers, neuroimaging, and neuropathology. Results: Of 956 individuals, 197 (20.6\%) had aPL positivity, defined as positivity on any of the assays, at the time of first measurement. During a mean follow-up of 6.6 years (SD 4), overall aPL positivity was not associated with change in global cognition (estimate $=-0.005, \mathrm{SE} 0.011$; $p=0.622$ ) or parkinsonian signs (estimate $=-0.003$, SE 0.017; $p=0.860)$. aPL were not associated with serum CRP or MMPs (both $p>0.268$ ). aPL were not associated with in vivo brain magnetic resonance imaging white matter hyperintensities or infarcts (both $p>0.376$ ). Among those autopsied, aPL were not associated with pathologically confirmed brain

\section{KARGER}

(c) 2019 S. Karger AG, Basel

E-Mail karger@karger.com

www.karger.com/ned
Zoe Arvanitakis, MD, MS

Rush Alzheimer's Disease Center

Rush University Medical Center

Chicago, IL 60612 (USA)

E-Mail zarvanit@ rush.edu 
infarcts, or cerebral atherosclerosis or arteriolosclerosis (all $p \geq 0.447$ ). Conclusions: In older individuals followed longitudinally, aPL do not relate to cognitive or motor decline, inflammation, or cerebrovascular disease on in vivo neuroimaging or postmortem neuropathology.

(c) 2019 S. Karger AG, Basel

\section{Introduction}

Cognitive and motor impairments in aging present a public heath challenge, given that they are common and associated with disability and mortality $[1,2]$. Yet, few good treatment options are available and prevention remains the most promising approach to address these problems. Identifying modifiable risk factors (e.g., vascular such as hypertension and diabetes), is a cornerstone in biomedical research on brain dysfunction in aging [3]. Antiphospholipid (aPL) antibodies are auto-antibodies directed against a cell membranes (phospholipids) in many tissues including brain, and easily measurable in blood [4]. These antibodies, such as anticardiolipin (aCL) antibodies, increase risk of ischemic stroke by twofold, usually in early to mid-adult life, and in specific patients (e.g., with systemic lupus erythematosus) $[5,6]$. Treatments for stroke prevention in persons with aPL are available, including antiplatelets or anticoagulants. Given that aPL have been associated with cognitive impairment in select patient groups or small studies, researchers recognize the need to systematically study aPL and brain function outcomes [7-9]. While aPL are known to increase in prevalence with aging [10], few data are available on the relation of aPL to cognitive and motor function in older persons, the 2 most common clinical manifestations of underlying cerebrovascular disease. There are preliminary data that suggest that aCL are associated with cognitive and motor function including parkinsonism $[11,12]$. However, we are not aware of any prior study on aPL and cognitive and motor decline in older persons without known syndromes associated with aPL.

The overall goal of the aPL Antibodies, Brain Infarcts, and Cognitive and Motor Decline in Aging study was to examine the association, in community-dwelling older persons, of aPL with brain infarcts and its 2 common consequences, decline in cognitive and motor function. The study design and methods are reported elsewhere [13]. We previously published, among 607 autopsied participants, that aPL were not associated with neuropathologically identified brain infarcts [14]. Here, we expand our prior study in several ways, by increasing the sample size and adding additional clinical, laboratory, and neuropathologic outcomes. The current study reports about nearly 1,000 community-dwelling participants from one of two ongoing cohort studies of aging, who followed annually for 7 years and who underwent autopsy at time of death. Using mixed effects analyses, we examined the relation of serum aPL to change in cognitive and motor function, including overall measures of global cognition and parkinsonian signs, and in secondary analyses, of 5 cognitive domains and additional motor measures. In secondary analyses among participant subgroups with additional data, we examined the associations of aPL to serum markers of inflammation, and to brain magnetic resonance imaging (MRI) measures of cerebrovascular disease (e.g., white matter hyperintensities). Among 699 autopsied persons, we reexamined the associations of aPL with brain infarcts, including analyses of infarct size and location, and with cerebral vessel disease outcomes including atherosclerosis and arteriolosclerosis.

\section{Methods}

Methods are described in the online supplementary Material (see www.karger.com/doi/10.1159/000500157).

\section{Participants and Data}

Participants from one of two ongoing, prospective, community-based, clinical-pathologic cohort studies of aging were included: the Religious Orders Study and the Rush Memory and Aging Project. Both have high follow-up and autopsy rates - above $85 \%$ [15]. Annual clinical evaluations include neuropsychological testing using a standardized battery, from which composite measures of 5 cognitive domains and an overall score of global cognition are derived [16]. Motor testing includes a modified version of the Unified Parkinson's Disease Rating Scale, yielding an overall score and 4 sign sub-scores [17]. Additionally, performance-based motor data are used to create a composite global motor score [18].

Clinical evaluations include a blood draw, from which aPL and other serum measures were quantified, blinded to all clinical data. Three serum aPL measures, each with IgG and IgM titers, were collected: aCL antibodies, antiphosphatidyl-serine (aPS) antibodies, and antibodies to $\beta_{2}$-glycoprotein I (anti- $\beta_{2}$ GPI) [13]. In a subset, longitudinal aPL data were collected. For analyses, we created a summary variable of overall aPL positivity (yes vs. no), if any of the aPL assays were positive according to the manufacturer's threshold. Additionally, serum markers of inflammation and blood brain barrier (BBB) permeability were also collected in the same sample: C-reactive protein (CRP) and matrix metalloproteinase 9 and matrix metalloproteinase tissue inhibitor, from which we created a ratio (MMPr) [19].

Brain MRI data were collected using a 1.5 Tesla General Electric (Waukesha, WI, USA) scanner, from which white matter hyperintensities total volume and infarcts with volume of $3^{3} \mathrm{~mm}^{3}$ or more were derived $[20,21]$. 
Postmortem neuropathologic examinations documented brain infarcts, including gross and microinfarcts, as well as vessel diseases $[15,22]$.

\section{Statistical Analysis}

To test the primary hypothesis, we used linear mixed effect models with overall aPL positivity at baseline as the predictor and change in the primary outcome variables of global cognitive function and global parkinsonian signs score. Secondary analyses used outcomes of 5 cognitive domains (perceptual speed, working memory, episodic memory, semantic memory, and visuospatial abilities) and parkinsonian signs sub-scores and the global motor score measure. To test the association of aPL outcomes on serum, neuroimaging, and neuropathology, we used linear regressions for continuous outcomes, logistic regressions for binary outcomes, and ordinal logistic regressions for ordinal level outcomes.

\section{Results}

Participant Characteristics and Laboratory Measures

Participant demographic, clinical, neuroimaging, and neuropathologic characteristics are shown in Table 1. The descriptive data on the panel of the 3 individual aPL measures, including for IgG and IgM separately for each, are provided (Table 2). Also, aPLs were measured over time in 463 out of 956 (48.4\%), and data suggested variability in aPL. A subset of 481 out of 953 participants had CRP and matrix metalloproteinases (MMPs) data. A subset of 413 had neuroimaging brain MRI data on white matter hyperintensities and 330 on infarcts (Table 1). Over the study course, 699 participants with aPL data died and were autopsied (Table 1).

\section{Relation of aPL to Level and Change in Cognitive and Motor Function}

The mean follow-up time interval from baseline (first aPL measurement) to time of analyses was 6.6 (SD 4.0) years. We conducted a series of mixed effects models, all adjusted for age, sex, and education, to examine the relation of baseline aPL to level and change in cognitive function. As shown in Table 3, the overall aPL positivity was not related to level $(p=0.203)$ or decline $(p=0.622)$ in the global cognitive score or any of the 5 cognitive domain measures (all $p>0.099$ ). Results were similar (no relation of aPL with the global cognitive score level or decline), when taking cohort effects into account (data not shown). We next conducted similar analyses but replacing the outcome variables with measures of motor function (Table 3). We found that aPL were associated with a higher level at baseline (worse score) in the global parkinsonian signs score $(p=0.047)$, as well as in secondary measures of gait disturbance and bradykinesia. There was a borderline significant association of aPL with a worse level on the global motor score. But, aPL were not related to change any of the motor function measures, including the primary measure of global parkinsonian signs score or any of the secondary measures (Table 3 ; all $p>0.532$ ). In logistic mixed effects models, similarly adjusted, there was no significant relation of overall aPL positivity with the presence at baseline or with incident parkinsonian signs of tremor or rigidity (all $p>0.088$ ).

\section{Relation of aPL to Laboratory (Serum) Markers of Inflammation and $B B B$}

Among 481 participants, linear regression analyses adjusted for the age, sex, and education, examined the relation of baseline overall aPL positivity to serum markers of inflammation and BBB permeability. In the analysis with $\mathrm{CRP}$ as the outcome, there was no association of overall aPL positivity with CRP $(p=0.268)$. In secondary analyses using predictors of individual aPL measures, aPS was associated with lower CRP ( $p=0.022$; not adjusted for multiple comparisons), but aCL and anti- $\beta_{2}$ GPI were not (both $p>0.269$ ). In the analysis with MMPr as the outcome, there was no association of overall aPL positivity with $\operatorname{MMPr}(p=0.583)$. In secondary analyses using predictors of individual aPL measures, aPS, aCL, and anti$\beta_{2}$ GPI were not associated with $\operatorname{MMPr}($ all $p>0.623)$. In addition, there was no association of any of the aPL measures with either of the 2 individual MMP sub-measures (data not shown).

\section{Relation of aPL to Neuroimaging Markers of Cerebrovascular Disease}

Among 413 participants, linear regression analyses adjusted for the age, sex, and education, examined the relation of baseline overall aPL positivity to brain MRI white matter hyperintensities. There was no association of any of the aPL measures (overall or individual measures) with white matter hyperintensities (all $p>0.376$ ). The ICCs for inter-rater and intra-rater reliability of infarct segmentation were 0.97 and 0.93 respectively. Using data from 330 persons, we found no association of overall aPL positivity with the presence of MRI infarcts $\left(\chi^{2}[1]=0.182\right.$, $p=0.670$ ), and the association remained nonsignificant in an adjusted logistic regression.

\section{Relation of aPL to Pathologic Markers of Cerebrovascular Disease}

Among 699 autopsied persons with neuropathologic data, we conducted regression analyses, adjusted for the 
Table 1. Characteristics of participants by aPL status at study baseline*

\begin{tabular}{|c|c|c|c|}
\hline Data & $\begin{array}{l}\text { aPL positivity on any } \\
\text { measure }(n=197)\end{array}$ & $\begin{array}{l}\text { aPL negativity on all } \\
\text { measures }(n=759)\end{array}$ & $\begin{array}{l}\text { Combined } \\
(n=956)\end{array}$ \\
\hline \multicolumn{4}{|l|}{ Demographic } \\
\hline Age at baseline, years, mean (SD) & $81.4(6.3)$ & $81.1(7.3)$ & $81.1(7.1)$ \\
\hline Women, $n(\%)$ & $140(71.1)$ & $548(72.2)$ & $688(72.0)$ \\
\hline Education, years, mean (SD) & $15.2(3.3)$ & $15.3(3.1)$ & $15.3(3.2)$ \\
\hline \multicolumn{4}{|l|}{ Cognitive** } \\
\hline Global cognitive function score & $-0.1(0.7)$ & $0.0(0.6)$ & $0.0(0.6)$ \\
\hline Perceptual speed score & $-0.3(0.9)$ & $-0.1(0.9)$ & $-0.1(0.9)$ \\
\hline Working memory score & $-0.1(0.7)$ & $0.1(0.8)$ & $0.1(0.7)$ \\
\hline Episodic memory score & $-0.1(0.9)$ & $0.0(0.8)$ & $0.0(0.8)$ \\
\hline Semantic memory score & $-0.1(0.8)$ & $0.0(0.7)$ & $0.0(0.7)$ \\
\hline Visuospatial ability score & $0.0(0.9)$ & $0.1(0.8)$ & $0.1(0.8)$ \\
\hline \multicolumn{4}{|l|}{ Motor** } \\
\hline Global parkinsonian signs score & $2.9(1.5)$ & $2.7(1.4)$ & $2.7(1.5)$ \\
\hline Gait disturbance score & $4.2(2.3)$ & $3.6(2.2)$ & $3.7(2.3)$ \\
\hline Bradykinesia score & $2.8(2.2)$ & $2.5(2.2)$ & $2.6(2.2)$ \\
\hline Rigidity score & $1.2(2.0)$ & $1.1(1.8)$ & $1.1(1.8)$ \\
\hline Tremor score & $1.0(1.5)$ & $1.0(1.4)$ & $1.0(1.4)$ \\
\hline Global motor score & $0.9(0.2)$ & $1.0(0.3)$ & $1.0(0.3)$ \\
\hline \multicolumn{4}{|l|}{ Laboratory } \\
\hline CRP & $3,610.3(5,314.4)$ & $4,573.7(7,309.8)$ & $4,409.0(7,013.5)$ \\
\hline MMP (ratio) & $1.9(25.6)$ & $-26.6(462.0)$ & $-21.8(420.9)$ \\
\hline \multicolumn{4}{|l|}{ Neuroimaging } \\
\hline White matter hyperintensity score & $-0.3(0.4)$ & $-0.3(0.4)$ & $-0.3(0.4)$ \\
\hline Any infarct, $n(\%)$ & $13 / 56(23)$ & $75 / 274(27)$ & $88 / 300(27)$ \\
\hline \multicolumn{4}{|l|}{ Neuropathologic, $n(\%)$} \\
\hline Any brain infarct present ${ }^{* * *}$ & $65(48)$ & $237(52)$ & $302(51)$ \\
\hline Gross infarct present & $49(36)$ & $168(37)$ & $217(36)$ \\
\hline Microinfarct present & $39(29)$ & $145(32)$ & $184(31)$ \\
\hline Cortical & $37(26)$ & $138(29)$ & $175(28)$ \\
\hline Subcortical & $48(34)$ & $174(36)$ & $222(36)$ \\
\hline \multicolumn{4}{|l|}{ Vessel pathology, $n(\%)^{* * * *}$} \\
\hline Atherosclerosis & $53(38)$ & $175(36)$ & $228(37)$ \\
\hline Arteriosclerosis & $47(33)$ & $162(34)$ & $209(34)$ \\
\hline \multicolumn{4}{|c|}{$\begin{array}{l}\text { * Mean (SD) at time of first aPL measure, unless otherwise specified; aPL positivity defined as positivity or } \\
\text { any of the } 6 \text { blood measures at baseline (IgG and IgM for each of the } 3 \mathrm{aPL} \text { ). } \\
\text { ** At study baseline. } \\
\text { *** Any infarct refers to infarcts of any size (gross or microscopic) and location (cortical or subcortical). } \\
\text { **** Grade of moderate-to-severe. } \\
\text { aPL, antiphospholipid; CRP, C-reactive protein; MMP, matrix metalloproteinases. }\end{array}$} \\
\hline
\end{tabular}

age, sex, and education, to examine the relation of baseline overall aPL positivity to cerebrovascular disease (Table 4). In logistic regression analyses, aPL were not associated with any of the infarct measures, including the presence of any infarct, or separately for gross- or microinfarcts, or infarcts in the cortical or subcortical regions (all $p>0.44$ ). Results were similar (no relation of aPL with any infarcts), when taking cohort effects into account (data not shown). Further, in ordinal logistic regression analyses, aPL was not associated with cerebral vessel disease severity, either in large or small vessels (Table 4). 


\section{Discussion}

In this study of nearly 1,000 older community-dwelling persons, serum aPL positivity was common, being present in one fifth of persons. In analyses using annual data over an average of 7 years, we did not find a relation of aPL positivity at baseline with level or decline in measures of global cognition or in 5 separate cognitive domains. We found associations of aPL with worse baseline levels of the global parkinsonian sign score and in parkinsonian sub-scores, but not with decline in any of the motor function outcomes. In additional cross-sectional analyses, there was no association of aPL with serum measures of inflammation or blood-brain barrier breakdown. We found no evidence for an association of aPL with in vivo MRI measures of cerebrovascular disease including white matter hyperintensities, nor with postmortem measures of cerebrovascular neuropathology including infarcts of any size or location, or cerebral vessel diseases affecting large or small vessels. In summary, this study did not find that aPL in older persons was related to cognitive or motor decline, or to plausible pathogenic mechanisms of ischemic stroke.

The frequency of aPL positivity increases with increasing age [10]. While rare at younger ages such as early and mid-adult life, aPL are usually observed in specific disease states (e.g., systemic lupus erythematosus, aPL antibody syndrome). Our data support the little data available in older and relatively healthy persons (without known aPLrelated syndromes), in particular the data from the Framingham Cohort and Offspring Study which found, in almost 5,000 middle aged persons (mean age $=59$ years) followed for 11 years, that aCL increases with age from $20 \%$ of persons in the 6 th decade to $35 \%$ in those $>80$ years [10]. Indeed, we found that aPL positivity in any of the $3 \mathrm{aPL}$ assayed was present in about $21 \%$ of persons with an average age of 81 years. The most common positive measure was for aCL, and in contrast to the Framingham data, we found aCL to be present in about $17 \%$ of participants in the 8th decade, about half as frequent as in the Framingham study. We add to the literature in older persons, by assaying IgG and IgM for $3 \mathrm{aPL}$ in a large group, and also by examining positivity over time in a subset. In about half of participants, we assessed aPL at a second or third time point, about 2 years apart in between each measure. We found variability in aPL positivity, with about $70 \%$ of those positive at the first time point remaining positive. Why positivity increases with age, and why positivity is variable over the years in older persons, remains unclear. Possible explanations include increased
Table 2. aPL positivity at baseline among 956 participants

\begin{tabular}{lc}
\hline Variable & aPL positivity, $n(\%)$ \\
\hline Overall aPL positivity & $197(20.6)$ \\
aCL antibodies & $165(17.3)$ \\
$\quad$ IgG & $43(4.5)$ \\
IgM & $126(13.2)$ \\
anti- $\beta_{2}$ GPI & $87(9.1)$ \\
IgG & $26(2.7)$ \\
IgM & $65(6.8)$ \\
aPS antibodies & $58(6.1)$ \\
IgG & $12(1.3)$ \\
IgM & $46(4.8)$ \\
\hline
\end{tabular}

aPL, antiphospholipid; aCL, anticardiolipin; anti- $\beta_{2} \mathrm{GPI}$, antibodies to $\beta_{2}$-glycoprotein I; aPS, antiphosphatidyl-serine.

autoimmune pathogenecity with aging (similar to increased thyroid disease), benign immune senescence including with increased circulating autoimmune markers without associated disease, and other factors as-of-yet to be defined [23]. Research will need to clarify why aPL increase with age and what factors contribute to its variability over time.

While several studies suggest that aPL are associated with cognitive impairment and decline, these are largely limited to younger adults, small samples of individuals, outpatient clinical settings, and select patients with specific disease states $[7,8,24]$. There are few studies on aPL and cognition and motor function in older adults. In a large stroke prevention cohort of nearly 2,000 older persons, Homayoon et al. [11] found that aCL was associated with a lower score on the Mini-Mental State Examination. In a small clinic-based study, aCL positivity was high among patients with vascular parkinsonism [12]. The finding of no relation with change in function, even in the presence of a level effect for motor function, has been found for other cardiovascular measures [25] but reduces the importance of aPL vis-a-vis brain function in aging. Indeed, while aPL are frequent in aging because they are not associated with decline in brain function (cognitive or motor function) and do not have a clear clinical significance, the currently used cutoffs for aPL positivity may need to be reevaluated for older persons.

Little data on relation of aPL to brain MRI are available in older persons [26]. In the recent study by Homayoon et al. [11], there was no relation of aCL with white matter lesions or infarcts on brain MRI among nearly 1,000 older persons. Our study is in keeping with those results, as 
Table 3. Relation of overall aPL positivity at baseline to level and change in cognitive and motor function $(n=$ 956)*

\begin{tabular}{|c|c|c|c|c|c|c|}
\hline \multirow[t]{2}{*}{ Outcome } & \multicolumn{3}{|c|}{ Level of function (at baseline) } & \multicolumn{3}{|c|}{ Change in function (over time) } \\
\hline & estimate & SE & $p$ value & estimate & SE & $p$ value \\
\hline \multicolumn{7}{|l|}{ Cognitive function } \\
\hline Global cognitive function score ${ }^{* *}$ & -0.062 & 0.048 & 0.203 & -0.005 & 0.011 & 0.622 \\
\hline Perceptual speed score & -0.102 & 0.069 & 0.138 & -0.012 & 0.012 & 0.306 \\
\hline Working memory score & -0.091 & 0.055 & 0.099 & -0.009 & 0.010 & 0.350 \\
\hline Episodic memory score & -0.036 & 0.064 & 0.581 & -0.005 & 0.012 & 0.679 \\
\hline Semantic memory score & -0.066 & 0.053 & 0.218 & -0.019 & 0.012 & 0.126 \\
\hline Visuospatial ability score & -0.034 & 0.057 & 0.552 & -0.009 & 0.009 & 0.320 \\
\hline \multicolumn{7}{|l|}{ Motor function } \\
\hline Global parkinsonian signs score** & 0.192 & 0.097 & 0.047 & -0.003 & 0.017 & 0.860 \\
\hline Gait disturbance score & 0.360 & 0.152 & 0.018 & -0.013 & 0.026 & 0.617 \\
\hline Bradykinesia score & 0.277 & 0.137 & 0.043 & -0.017 & 0.028 & 0.532 \\
\hline Global motor score & -0.031 & 0.017 & 0.064 & -0.001 & 0.002 & 0.823 \\
\hline
\end{tabular}

* All 10 mixed effects models (one per row) were adjusted for age, sex, and education.

** Primary outcome measure.

aPL, antiphospholipid.

Table 4. Relation of overall aPL positivity at baseline to cerebrovascular disease on postmortem neuropathology $(n=699)^{*}$

\begin{tabular}{llcll}
\hline Infarct outcome & OR & Estimate & SE & $p$ value \\
\hline Presence of any brain infarcts** & 1.007 & 0.007 & 0.183 & 0.971 \\
$\quad$ Gross infarcts & 1.113 & 0.107 & 0.189 & 0.570 \\
$\quad$ Microinfarcts & 0.906 & -0.099 & 0.201 & 0.623 \\
$\quad$ Cortical infarcts & 0.854 & -0.158 & 0.208 & 0.447 \\
$\quad$ Subcortical infarcts & 1.026 & 0.026 & 0.191 & 0.893 \\
Atherosclerosis*** & 1.103 & 0.098 & 0.169 & 0.560 \\
Arteriolosclerosis*** & 1.122 & 0.116 & 0.167 & 0.490 \\
\hline
\end{tabular}

* All regression models adjusted for age, sex, and education.

** Logistic regression analyses.

*** Ordinal logistic regression, assuming proportional odds.

aPL, antiphospholipid.

we did not find associations of aPL with in vivo MRI measures of white matter hyperintensities or infarcts. We are aware of only one prior large study, and by our group and using many of the same participants as here, which systematically examined aPL and cerebrovascular disease by neuropathology, and results were null [14]. Building on our prior publication, we confirm the null finding of aPL with postmortem measures of any infarcts, including by size (gross and micro-infarcts) and location (cortical and subcortical). We expand the data by examining the association of aPL with severity of cerebral vessel diseases, specifically atherosclerosis and arteriolosclerosis, and found no associations. Because other pathogenic processes may be involved in persons with aPL positivity, we also examined other serum measures sometimes altered in brain disease states. We did not find evidence for associations of aPL with markers of inflammation (serum CRP) or blood-brain barrier permeability breakdown (serum MMPs). Taken together, our results are consistent in not supporting a relation of aPL with cerebrovascular disease in older persons, whether by neuroimaging or neuropathology measures. 
Several strengths of this study are worth mentioning. First, we assayed a total of 6 aPL measures in a very large group of persons, and over time in half the persons, using commercially available assay kits, which are wellvalidated, widely available, and relatively inexpensive. Second, we used primary outcome measures of global cognitive and motor function, which decrease ceiling effects in relatively healthy persons and which have been well-validated in these and other cohorts $[15,27]$. We further used cognitive domains and other motor score outcomes as secondary measures, which allowed us to examine for possible differential effects within systems and for consistency of findings across the systems. Third, given the high follow-up rate of the cohorts, longitudinal data on cognitive and motor function were very complete, and provides high internal validity of this study. Fourth, we assessed a range of in vivo and postmortem measures of cerebrovascular disease, all collected blinded to clinical data (including to cognitive and motor function), thus providing a comprehensive examination of the question and further supporting the null finding.

This study has several important weaknesses. Most importantly, confidence in null findings are limited by several factors, such as whether a study has sufficient power to test the hypothesis, whether measurements were truly reflective of the processes of interest, whether research biases influenced the findings (e.g., information bias), among others. However, we took several precautions to avoid these and other threats to the validity of our results, including using a robust study design based on an observational longitudinal study with annually repeated measures, evaluating for and using the necessary sample size to reach adequate power to test our hypothesis [13], and using several complementary measures for each of the main variables of interest, specifically for aPL and cognitive and motor function, including by objective methods (e.g., assays performed blinded to clinical data; performance-based clinical measures of cognitive and motor function). These factors provide high-level confidence in our null results and other studies will need to replicate our findings. Nonetheless, for secondary measures of interest in this study, we only had data on a subset of persons, thus introducing the potential for inadequate power and information and other sources of biases. Indeed, only half of persons had data on serum CRP and MMPs, and about half or less had MRI data on the measures of cerebrovascular disease.

\section{Acknowledgments}

Authors wish to thank the participants of the Religious Orders Study and the Rush Memory and Aging Project, and the Rush Alzheimer's Disease Center research staff including the study managers, study coordinators, nurses, phlebotomists, laboratory technicians, research assistants, and data and analytic programmers.

\section{Disclosure Statement}

Authors report that there are no conflicts of interest to disclose.

\section{Funding Source}

This study was supported by the National Institutes of Health grant numbers P30AG010161, RF1AG015819, R01AG17917, R01AG40039, and R01NS084965.

\section{References}

1 Mokdad AH, Ballestros K, Echko M, Glenn S, Olsen HE, Mullany E, et al.; US Burden of Disease Collaborators. The State of US Health, 1990-2016: Burden of Diseases, Injuries, and Risk Factors Among US States. JAMA. 2018 Apr;319(14):1444-72.

2 Fried LP, Ferrucci L, Darer J, Williamson JD, Anderson G. Untangling the concepts of disability, frailty, and comorbidity: implications for improved targeting and care. J Gerontol A Biol Sci Med Sci. 2004 Mar; 59(3):255-63.

3 Corriveau RA, Bosetti F, Emr M, Gladman JT, Koenig JI, Moy CS, et al. The Science of Vascular Contributions to Cognitive Impairment and Dementia (VCID): A Framework for Advancing Research Priorities in the Cerebrovascular Biology of Cognitive Decline. Cell Mol Neurobiol. 2016 Mar;36(2): 281-8.

4 Shapiro SS, Thiagarajan P, De Marco L. Mechanism of action of the lupus anticoagulant. Ann N Y Acad Sci. 1981;370(1):359-65.

5 Levine SR, Brey RL, Tilley BC, Thompson JL, Sacco RL, Sciacca RR, et al.; APASS Investigators. Antiphospholipid antibodies and subsequent thrombo-occlusive events in patients with ischemic stroke. JAMA. 2004 Feb;291(5): 576-84.

6 Cervera R, Serrano R, Pons-Estel GJ, CeberioHualde L, Shoenfeld Y, de Ramón E, et al.; Euro-Phospholipid Project Group (European
Forum on Antiphospholipid Antibodies). Morbidity and mortality in the antiphospholipid syndrome during a 10 -year period: a multicentre prospective study of $1000 \mathrm{pa}$ tients. Ann Rheum Dis. 2015 Jun;74(6):10118.

7 Menon S, Jameson-Shortall E, Newman SP, Hall-Craggs MR, Chinn R, Isenberg DA. A longitudinal study of anticardiolipin antibody levels and cognitive functioning in systemic lupus erythematosus. Arthritis Rheum. 1999 Apr;42(4):735-41.

8 McLaurin EY, Holliday SL, Williams P, Brey RL. Predictors of cognitive dysfunction in patients with systemic lupus erythematosus. Neurology. 2005 Jan;64(2):297-303. 
9 Miyakis S, Lockshin MD, Atsumi T, Branch DW, Brey RL, Cervera R, et al. International consensus statement on an update of the classification criteria for definite antiphospholipid syndrome (APS). J Thromb Haemost. 2006 Feb;4(2):295-306.

10 Janardhan V, Wolf PA, Kase CS, Massaro JM, D’Agostino RB, Franzblau C, et al. Anticardiolipin antibodies and risk of ischemic stroke and transient ischemic attack: the Framingham cohort and offspring study. Stroke. 2004 Mar;35(3):736-41.

11 Homayoon N, Schwingenschuh P, Hofer E, Katschnig-Winter P, Schmidt R. Anticardiolipin antibodies are associated with cognitive dysfunction in stroke-free individuals. Eur J Neurol. 2014 Mar;21(3):427-432, e21-2.

12 Huang Z, Jacewicz M, Pfeiffer RF. Anticardiolipin antibody in vascular parkinsonism. Mov Disord. 2002 Sep;17(5):992-7.

13 Arvanitakis Z, Brey RL, Rand JH, Schneider JA, Leurgans SE, Yu L, et al. Antiphospholipid antibodies, brain infarcts, and cognitive and motor decline in aging (ABICMA): design of a community-based, longitudinal, clinical-pathological study. Neuroepidemiology. 2013;40(2):73-84.

14 Arvanitakis Z, Brey RL, Rand JH, Schneider JA, Capuano AW, Yu L, et al. Relation of antiphospholipid antibodies to postmortem brain infarcts in older people. Circulation. 2015 Jan;131(2):182-9.

15 Bennett DA, Buchman AS, Boyle PA, Barnes LL, Wilson RS, Schneider JA. Religious Or- ders Study and Rush Memory and Aging Project. J Alzheimers Dis. 2018;64(s1):S16189.

16 Barnes LL, Yumoto F, Capuano A, Wilson RS, Bennett DA, Tractenberg RE. Examination of the Factor Structure of a Global Cognitive Function Battery across Race and Time. J Int Neuropsychol Soc. 2016 Jan; 22(1):66-75.

17 Arvanitakis Z, Wilson RS, Schneider JA, Bienias JL, Evans DA, Bennett DA. Diabetes mellitus and progression of rigidity and gait disturbance in older persons. Neurology. 2004 Sep;63(6):996-1001.

18 Buchman AS, Leurgans SE, Yu L, Wilson RS, Lim AS, James BD, et al. Incident parkinsonism in older adults without Parkinson disease. Neurology. 2016 Sep;87(10):103644.

19 Karabudak R, Kurne A, Guc D, Sengelen M, Canpinar H, Kansu E. Effect of interferon beta-1a on serum matrix metalloproteinase- 9 (MMP-9) and tissue inhibitor of matrix metalloproteinase (TIMP-1) in relapsing remitting multiple sclerosis patients. One year follow-up results. J Neurol. 2004 Mar;251(3): 279-83.

20 Arvanitakis Z, Fleischman DA, Arfanakis K, Leurgans SE, Barnes LL, Bennett DA. Association of white matter hyperintensities and gray matter volume with cognition in older individuals without cognitive impairment. Brain Struct Funct. 2016 May;221(4):213546.
21 Zacharaki EI, Kanterakis S, Bryan RN, Davatzikos $C$. Measuring brain lesion progression with a supervised tissue classification system. Med Image Comput Comput Assist Interv. 2008;11(Pt 1):620-627.

22 Arvanitakis Z, Capuano AW, Leurgans SE, Buchman AS, Bennett DA, Schneider JA. The Relationship of Cerebral Vessel Pathology to Brain Microinfarcts. Brain Pathol. 2017 Jan; 27(1):77-85

23 Nikolich-Žugich J. The twilight of immunity: emerging concepts in aging of the immune system. Nat Immunol. 2018 Jan;19(1):10-9.

24 Conti F, Alessandri C, Perricone C, Scrivo R, Rezai S, Ceccarelli F, et al. Neurocognitive dysfunction in systemic lupus erythematosus: association with antiphospholipid antibodies, disease activity and chronic damage. PLoS One. 2012;7(3):e33824.

25 Boyle PA, Yang J, Yu L, Leurgans SE, Capuano AW, Schneider JA, et al. Varied effects of age-related neuropathologies on the trajectory of late life cognitive decline. Brain. 2017 Mar;140(3):804-12.

26 Schmidt R, Auer-Grumbach P, Fazekas F, Offenbacher H, Kapeller P. Anticardiolipin antibodies in normal subjects. Neuropsychological correlates and MRI findings. Stroke. 1995 May;26(5):749-54.

27 Buchman AS, Wilson RS, Yu L, Boyle PA, Bennett DA, Barnes LL. Motor Function Is Associated With Incident Disability in Older African Americans. J Gerontol A Biol Sci Med Sci. 2016 May;71(5):696-702. 